\title{
Leptospira Exposure and Gardeners: A Case-Control Seroprevalence Study
}

\author{
Cosme Alvarado-Esquivele ${ }^{\mathrm{a}, \mathrm{d}}$, Jesus Hernandez-Tinoco ${ }^{\mathrm{b}}$, Luis Francisco Sanchez-Anguiano ${ }^{\mathrm{b}}$, \\ Agar Ramos-Nevarez ${ }^{\mathrm{c}}$, Sandra Margarita Cerrillo-Soto ${ }^{\mathrm{c}}$, Carlos Alberto Guido-Arreola ${ }^{\mathrm{c}}$
}

\begin{abstract}
Background: Leptospira can be found in soil. However, it is unclear whether occupational exposure to soil may represent a risk for Leptospira infection in humans. Therefore, we sought to determine the association of Leptospira IgG seroprevalence with the occupation of gardener, and to determine the epidemiological characteristics of gardeners associated with Leptospira exposure.
\end{abstract}

Methods: We performed a case-control study in 168 gardeners and 168 age- and gender-matched control subjects without gardening occupation in Durango City, Mexico. The seroprevalence of anti-Leptospira IgG antibodies in cases and controls was determined using an enzyme immunoassay. Bivariate and multivariate analyses were used to assess the association of Leptospira exposure and the characteristics of the gardeners.

Results: Anti-Leptospira IgG antibodies were found in $10(6 \%)$ of 168 gardeners and in $15(8.9 \%)$ of 168 control subjects (odds ratio (OR): $0.64 ; 95 \%$ confidence interval $(\mathrm{CI}): 0.28-1.48 ; \mathrm{P}=0.40$ ). Multivariate analysis showed that Leptospira seropositivity was positively associated with female gender (OR: 5.82; 95\% CI: 1.11 - 30.46; $\mathrm{P}=0.03$ ), and negatively associated with eating while working (OR: $0.21 ; 95 \% \mathrm{CI}: 0.05-0.87 ; \mathrm{P}=0.03)$. In addition, multivariate analysis showed that high anti-Leptospira levels were associated with consumption of boar meat (OR: 28.00; 95\% CI: 1.20 - 648.80; P = 0.03).

Conclusions: This is the first case-control study of Leptospira exposure in gardeners. Results do not support an association of Leptospira exposure with the occupation of gardener. However, further studies to confirm the lack of this association are needed. The potential role

Manuscript accepted for publication October 23, 2015

aBiomedical Research Laboratory, Faculty of Medicine and Nutrition, Juarez University of Durango State, Durango, Mexico

bInstitute for Scientific Research "Dr. Roberto Rivera Damm", Juarez University of Durango State, Durango, Mexico

${ }^{\mathrm{c} C l i n i c a}$ de Medicina Familiar, Instituto de Seguridad y Servicios Sociales de los Trabajadores del Estado, Predio Canoas S/N, 34079 Durango, Mexico

${ }^{\mathrm{d} C}$ Corresponding Author: Cosme Alvarado-Esquivel, Faculty of Medicine and

Nutrition, Av. Universidad S/N, 34000 Durango, Mexico.

Email: alvaradocosme@yahoo.com

doi: http://dx.doi.org/10.14740/jocmr2377w of consumption of boar meat in Leptospira infection deserves further investigation.

Keywords: Leptospira; Epidemiology; Seroprevalence; Gardeners; Case-control study; Mexico

\section{Introduction}

The pathogenic bacteria of the genus Leptospira cause morbidity and mortality worldwide [1]. Leptospirosis, the disease caused by Leptospira, is considered the most widespread zoonosis in the world [2]. The higher incidence of leptospirosis occurs in the tropics and subtropics [3]. Humans become infected with Leptospira by contact with Leptospira-infected animals or their urine [3, 4]. Evidence of Leptospira infection in virtually all mammalian species examined has been found [2]. Infection can also be acquired from the environment contaminated with Leptospira, i.e., soil and water [2, 5, 6]. Leptospirosis outbreaks have been associated with common water events including occupational exposure, water sports, consumption of water, and water disasters [7]. Infection with Leptospira occurs through skin abrasions [8]. The clinical spectrum of Leptospira infection varies from subclinical to life-threatening disease [9]. Clinical presentation of leptospirosis includes fever, flu-like symptoms, headache, intense myalgia, and liver involvement with jaundice and kidney involvement $[8,9]$.

Leptospira shed in the urine of infected animals may contaminate soil of gardens. These places are often visited by domestic and wild animals that may be infected with Leptospira, i.e., rats [1, 10, 11], dogs [12, 13], and cats [14]. Gardeners have a frequent contact with water and soil. Untreated water (sometimes from rivers or lakes) is used to water trees and plants in gardens in Durango, Mexico. Gardeners occasionally suffer from skin abrasions while working. Therefore, gardeners might have a risk for Leptospira infection. However, to the best of our knowledge, the association of Leptospira exposure with gardener occupation has not been studied. Therefore, this study aimed to determine the association of Leptospira IgG seropositivity and gardener occupation in Durango City, Mexico. The socio-demographic, work, clinical, and behavioral characteristics of the gardeners exposed to Leptospira were also investigated. 


\section{Materials and Methods}

\section{Study design and study populations}

This serosurvey has an age- and gender-matched case-control study design. We used residual serum samples from Toxoplasma gondii serosurveys in gardeners [15] and general population [16] in Durango City, Mexico. The proportion of cases and controls was 1:1. Thus, cases included 168 gardeners, and controls included 168 subjects of the general population without gardener occupation. Participants were studied for the presence of anti-Leptospira IgG antibodies. Inclusion criteria for gardeners were: 1) subjects who have been working as gardeners for at least 6 months in Durango City, Mexico; 2) aged 16 years and older; 3 ) any gender; and 4) who accepted to participate in the study. Socioeconomic status, or work place (private or government) were not restrictive criteria for enrollment. As a strategy to enroll gardeners, they were visited at their work places in streets, boulevards, parks and work offices. Gardeners were 16- 73 (mean: $38.15 \pm 13.91$ ) years old, 15 were females and 153 were males. The control group included 168 subjects randomly selected from the general population of Durango City matched with cases by age and gender. Thus, the control group included 15 females and 153 males aged 38.10 \pm 14.00 (range: 16 - 73) years old. No difference in age among cases and controls was found $(\mathrm{P}=0.97)$.

\section{General characteristics of gardeners}

Socio-demographic, work, clinical, and behavioral characteristics of gardeners were obtained from archival questionnaires [15]. Socioeconomic data included age, gender, birthplace, and educational level. In addition, the type of flooring at home was obtained. Work data included duration (years) in the activity, frequency of contact with water, sewage and soil, history of splashes at face with water, eating while working, washing hands before eating, history of injuries with sharp material at work, and use of safety practices (hand gloves, face masks and glasses). Clinical data included health status, history of blood transfusion or transplant history, and impairments in memory, reflexes, hearing and vision. Behavioral data included animal contacts, consumption of meat and type of meat consumed, degree of meat cooking, consumption of untreated water, unpasteurized milk or unwashed vegetables and fruits, eating away from home (in restaurants or fast food outlets), and traveling.

\section{Detection of Leptospira antibodies in serum}

Serum samples of gardeners and controls were analyzed for detection of anti-Leptospira IgG antibodies using a commercially available enzyme-linked immunosorbent assay kit, "Leptospira IgG ELISA test" (Diagnostic Automation Inc., Calabasas, CA). According to the kit's insert, this assay has a sensitivity of $100 \%$ and a specificity of $100 \%$. An absorb- ance reading of $\geq 0.5$ optical density (OD) units was used as a cut-off for positivity of anti-Leptospira IgG antibodies. High levels of anti-Leptospira IgG antibodies in sera were considered when absorbance readings $>1.0$ OD units were obtained. All tests were performed according to the instructions of the manufacturer, and positive and negative controls were included in each run.

\section{Statistical analysis}

Data were analyzed using the software Epi Info version 7 and SPSS version 15.0. Frequencies between groups were compared with the Fisher's exact test. Age among groups was compared with the Student's $t$-test. The association between the characteristics of gardeners and Leptospira exposure was assessed by bivariate and multivariate analyses. Variables with $P$ values $<0.10$ obtained in the bivariate analysis were selected for inclusion in the multivariate analysis. Logistic regression analysis with the Enter method was used to calculate the odds ratios (ORs) and 95\% confidence intervals (CIs). A P value < 0.05 was considered statistically significant. Two multivariate analysis models were used: one to assess the association of Leptospira exposure with the gardeners' characteristics, and other to assess the association of high anti-Leptospira antibody levels with the gardeners' characteristics. The Hosmer-Lemeshow goodness of fit test was used to assess the fitness of our regression models.

\section{Ethical aspects}

We studied only archival sera and data of participants from previous surveys $[15,16]$. This study was approved by the Ethical Committee of the Instituto de Seguridad y Servicios Sociales de los Trabajadores del Estado in Durango City. We obtained a written informed consent from major participants and from the next of kin of minor participants.

\section{Results}

Anti-Leptospira IgG antibodies were found in $10(6 \%)$ of 168 gardeners and in $15(8.9 \%)$ of 168 control subjects. No statistically significant difference in Leptospira seroprevalence among cases and controls was found (OR: 0.64; 95\% CI: 0.28 - 1.48; $\mathrm{P}=0.40)$. The frequency of high levels ( $\geq 1.0 \mathrm{OD}$ units) of anti-Leptospira IgG antibodies in gardeners (3/168: 1.8\%) was also similar to that (4/168: $2.4 \%$ ) in control subjects (OR: $0.74 ; 95 \%$ CI: $0.16-3.38 ; \mathrm{P}=1.00)$. Stratification by gender showed that three $(20 \%)$ of 15 female cases and null of $15 \mathrm{fe}-$ male controls were positive for anti-Leptospira antibodies $(\mathrm{P}=$ $0.22)$. On the other hand, seven $(4.6 \%)$ of 153 male cases and $15(9.8 \%)$ of 153 male controls were positive for anti-Leptospira antibodies $(\mathrm{P}=0.11)$.

Of the socio-demographic characteristics of gardeners, bivariate analysis showed that Leptospira exposure was associated with gender. Female gardeners had a significantly higher 
seroprevalence of Leptospira infection than male gardeners did (20\% vs. $4.6 \%$, respectively: $\mathrm{P}=0.04)$. Other socio-demographic characteristics of gardeners including age, birthplace, and education, and type of flooring at home showed $\mathrm{P}$ values $\geq$ 0.10 by bivariate analysis.

With respect to work characteristics of gardeners, two variables showed $\mathrm{P}$ values $<0.10$ by bivariate analysis: eating while working $(\mathrm{P}=0.03)$, and frequency of contact with water $(\mathrm{P}=0.09)$. Other work characteristics of gardeners including duration in the activity, frequency of contact with sewage and soil, history of splashes at face with water, washing hands before eating, history of injuries with sharp material at work, and use of safety practices showed $\mathrm{P}$ values $\geq 0.10$ by bivariate analysis.

None of the clinical characteristics including health status, history of blood transfusion or transplant history, and impairments in memory, reflexes, hearing and vision was associated with Leptospira exposure by bivariate analysis. Concerning behavioral characteristics of gardeners, the variable consumption of boar meat showed a $\mathrm{P}$ value of 0.06 by bivariate analysis. Whereas the variables including animal contacts, consumption of meat other than boar meat, degree of meat cooking, consumption of untreated water, unpasteurized milk or unwashed vegetables and fruits, eating away from home, and traveling showed $\mathrm{P}$ values $\geq 0.10$ by bivariate analysis. Multivariate analysis of the four variables (gender, eating while working, frequency of contact with water, and consumption of boar meat) with $\mathrm{P}$ values $<0.10$ obtained in the bivariate analysis showed that Leptospira seroreactivity was positively associated with female gender (OR: 5.82; 95\% CI: 1.11 - 30.46; P $=0.03)$, and negatively associated with eating while working (OR: $0.21 ; 95 \%$ CI: $0.05-0.87 ; \mathrm{P}=0.03$ ). The result of the Hosmer-Lemeshow test $(\mathrm{P}=0.61)$ suggested a good fitting of this regression model.

Bivariate analysis of the association of the gardeners' characteristics and high ( $>1.0$ OD units) anti-Leptospira IgG antibody levels showed three variables with $\mathrm{P}$ values $<0.10$ : consumption of boar meat $(\mathrm{P}=0.02)$, national trips $(\mathrm{P}=0.05)$, and consumption of sheep meat $(\mathrm{P}=0.07)$. Multivariate analysis of these characteristics showed that high ( $>1.0$ OD units) anti-Leptospira IgG antibody levels were associated only with consumption of boar meat (OR: 28.00; 95\% CI: 1.20 - 648.80; $\mathrm{P}=0.03)$. The result of the Hosmer-Lemeshow test $(\mathrm{P}=1.00)$ suggested a good fitting of this regression model.

\section{Discussion}

The seroepidemiology of Leptospira infection in workers occupationally exposed to soil is largely unknown. We are not aware of any report of descriptive or case-control studies on the seroprevalence of Leptospira infection in gardeners. Therefore, this study aimed to determine whether gardeners are at a higher risk for Leptospira infection than subjects without this occupation are. We found that gardeners had a similar seroprevalence of Leptospira exposure to controls. This result was unexpected since gardeners have a number of known risk factors for Leptospira exposure including contact with soil $[5,8]$, and water $[2,7]$. In addition, many gardeners studied did not use safety practices as wearing gloves, facemasks or glasses for avoiding infection. Skin abrasion is an important route of Leptospira infection [8]. However, injuries at work with sharp materials were not associated with Leptospira exposure in gardeners in our study. Furthermore, a considerable number of animals that may contaminate soil with their urine frequently visit gardens. The seroprevalence of Leptospira infection found in gardeners $(6 \%)$ is also comparable with seroprevalences reported in waste pickers (4.4\%) and their controls $(5.6 \%)$ in the same Durango City [17]. In contrast, the seroprevalence found in gardeners is lower than the $15.6 \%$ seroprevalence reported in general population in rural Durango [18]. Therefore, results suggest that gardeners do not have an increased risk for Leptospira exposure. The lack of studies about the epidemiology of Leptospira infection in gardeners does not allow us to compare our results with others, and additional studies to confirm or challenge our results are needed. It is unclear why Leptospira seropositivity was not associated with gardener occupation. Many animals urinate in gardens, and the number of animals with this practice may be higher in public gardens than in house gardens. Many of the gardeners studied worked in parks where dogs and other animals are seen daily. Rodents are considered the most important reservoir of Leptospira infection $[4,8,11]$. Thus, it is possible that the number of rodents in work places of gardeners was low, or the frequency of Leptospira infection in these animals was null or too low. It is also possible that the frequency of Leptospira infection in animals other than rodents living in or visiting the gardens was quite low, and thus soil contamination with Leptospira through urine of animals was low too. We are not aware of any study of Leptospira infection in animals in Durango, and the prevalence of this infection in rodents, dogs and other animals in Durango is largely unknown.

Analysis of the association of Leptospira seropositivity and gardeners' characteristics showed that Leptospira exposure was positively associated with female gender and negatively associated with eating while working. Leptospira seroprevalence has been associated with male gender [19, 20]. However, to the best of our knowledge, this is the first study that found an association of Leptospira seropositivity and female gender. It is unclear why female gardeners had a higher seroprevalence than male gardeners did. It is possible that female gardeners had different risk factors for Leptospira infection than male gardeners did. However, the number of females studied $(n=15)$ was low to perform a more extensive analysis of risk factors. Most gardeners in Durango City are males, and this was the reason we included more male gardeners than female gardeners in our study. On the other hand, the negative association of Leptospira exposure with eating while working suggests that this practice had no importance for Leptospira exposure among gardeners. Interestingly, multivariate analysis showed that high anti-Leptospira levels were associated with consumption of boar meat. We are not aware of any seroepidemiology study that had reported this association. However, infections with Leptospira have been demonstrated in boars. Leptospira DNA was found in kidneys of boars in Japan [21]. In addition, anti-Leptospira antibodies were found in $65.4 \%$ of wild boars in Portugal [22], and in 33\% of feral hogs (Sus scrofa) in Florida, USA [23]. Results of the epidemiological 
link between consumption of boar meat and Leptospira exposure found in this study warrant for further research.

\section{Conclusions}

This is the first case-control study of Leptospira exposure in gardeners. Results do not support an association of Leptospira exposure with the occupation of gardener. However, further research to confirm the lack of this association is needed. The potential role of the consumption of boar meat in Leptospira infection in humans deserves further investigation.

\section{Conflict of Interest}

None.

\section{Financial Support}

This study was financially supported by Juarez University of Durango State, Durango, Mexico.

\section{References}

1. Himsworth CG, Parsons KL, Jardine C, Patrick DM. Rats, cities, people, and pathogens: a systematic review and narrative synthesis of literature regarding the ecology of rat-associated zoonoses in urban centers. Vector Borne Zoonotic Dis. 2013;13(6):349-359.

2. Adler B, de la Pena Moctezuma A. Leptospira and leptospirosis. Vet Microbiol. 2010;140(3-4):287-296.

3. Guerra MA. Leptospirosis: public health perspectives. Biologicals. 2013;41(5):295-297.

4. Mayer-Scholl A, Hammerl JA, Schmidt S, Ulrich RG, Pfeffer M, Woll D, Scholz HC, et al. Leptospira spp. in rodents and shrews in Germany. Int J Environ Res Public Health. 2014;11(8):7562-7574.

5. Saito M, S YAMV, Masuzawa T, Yanagihara Y, Yoshida $\mathrm{S}$. [Leptospirosis now-the centennial of the discovery of Weil's disease pathogen]. Nihon Saikingaku Zasshi. 2014;69(4):589-600.

6. Wasinski B. [Leptospirosis--current problems]. Przegl Epidemiol. 2011;65(3):471-476.

7. Wynwood SJ, Graham GC, Weier SL, Collet TA, McKay DB, Craig SB. Leptospirosis from water sources. Pathog Glob Health. 2014;108(7):334-338.

8. Abgueguen P, Pichard E. [Leptospirosis]. Rev Prat. 2009;59(5):665-673.

9. van de Weyer RW, Ramakers BP, Pickkers P. [Leptospirosis]. Ned Tijdschr Geneeskd. 2015;159:A7797.

10. Costa F, Wunder EA, Jr., De Oliveira D, Bisht V, Rodrigues $\mathrm{G}$, Reis $\mathrm{MG}$, Ko AI, et al. Patterns in Leptospira Shedding in Norway Rats (Rattus norvegicus) from Bra- zilian Slum Communities at High Risk of Disease Transmission. PLoS Negl Trop Dis. 2015;9(6):e0003819.

11. Dupouey J, Faucher B, Edouard S, Richet H, Kodjo A, Drancourt M, Davoust B. Human leptospirosis: an emerging risk in Europe? Comp Immunol Microbiol Infect Dis. 2014;37(2):77-83.

12. Lelu M, Munoz-Zanzi C, Higgins B, Galloway R. Seroepidemiology of leptospirosis in dogs from rural and slum communities of Los Rios Region, Chile. BMC Vet Res. 2015;11:31.

13. Benkirane A, Noury S, Hartskeerl RA, Goris MG, Ahmed A, Nally JE. Preliminary Investigations on the Distribution of Leptospira Serovars in Domestic Animals in North-west Morocco. Transbound Emerg Dis. 2014.

14. Rodriguez J, Blais MC, Lapointe C, Arsenault J, Carioto L, Harel J. Serologic and urinary PCR survey of leptospirosis in healthy cats and in cats with kidney disease. J Vet Intern Med. 2014;28(2):284-293.

15. Alvarado-Esquivel C, Liesenfeld O, Marquez-Conde JA, Estrada-Martinez S, Dubey JP. Seroepidemiology of infection with Toxoplasma gondii in workers occupationally exposed to water, sewage, and soil in Durango, Mexico. J Parasitol. 2010;96(5):847-850.

16. Alvarado-Esquivel C, Estrada-Martinez S, Pizarro-Villalobos H, Arce-Quinones M, Liesenfeld O, Dubey JP. Seroepidemiology of Toxoplasma gondii infection in general population in a northern Mexican city. J Parasitol. 2011;97(1):40-43.

17. Alvarado-Esquivel C, Hernandez-Tinoco J, Sanchez-Anguiano LF, Ramos-Nevarez A, Cerrillo-Soto SM, GuidoArreola CA. Leptospira Exposure and Waste Pickers: A Case-Control Seroprevalence Study in Durango, Mexico. J Clin Med Res. 2015;7(8):637-640.

18. Alvarado-Esquivel C, Sanchez-Anguiano LF, HernandezTinoco J. Seroepidemiology of Leptospira Exposure in General Population in Rural Durango, Mexico. Biomed Res Int. 2015;2015:460578.

19. Ferro BE, Rodriguez AL, Perez M, Travi BL. [Seroprevalence of Leptospira infection in habitants of peripheral neighborhoods in Cali, Colombia]. Biomedica. 2006;26(2):250-257.

20. Everard CO, Hayes RJ, Edwards CN. Leptospiral infection in school-children from Trinidad and Barbados. Epidemiol Infect. 1989;103(1):143-156.

21. Koizumi N, Muto M, Yamamoto S, Baba Y, Kudo M, Tamae Y, Shimomura K, et al. Investigation of reservoir animals of Leptospira in the northern part of Miyazaki Prefecture. Jpn J Infect Dis. 2008;61(6):465-468.

22. Vale-Goncalves HM, Cabral JA, Faria MC, Nunes-Pereira M, Faria AS, Veloso O, Vieira ML, et al. Prevalence of Leptospira antibodies in wild boars (Sus scrofa) from Northern Portugal: risk factor analysis. Epidemiol Infect. 2015;143(10):2126-2130.

23. Chatfield J, Milleson M, Stoddard R, Bui DM, Galloway R. Serosurvey of leptospirosis in feral hogs (Sus scrofa) in Florida. J Zoo Wildl Med. 2013;44(2):404-407. 